\title{
Electronic Reliability Analysis under Radiation Environment
}

\author{
Cher Ming Tan, $, 2,3^{*}$ Vimal Kant Pandey, ${ }^{1}$ Yueh Chiang,,${ }^{1,4}$ and Tsung Ping Lee ${ }^{5}$ \\ ${ }^{1}$ Center for Reliability Science and Technology, Chang Gung University, \\ 259 Wen Hua 1st Road, Kwei-Shan, Taoyuan City 333323, Taiwan \\ ${ }^{2}$ Department of Urology, Chang Gung Memorial Hospital, Linkou, \\ No. 5, Fuxing Street, Guishan District, Taoyuan City 333423, Taiwan \\ ${ }^{3}$ Center for Reliability Engineering, Mingchi University of Technology, \\ No. 84, Gongzhuan Road, Taishan District, New Taipei City 243303, Taiwan \\ ${ }^{4}$ Department of Medical Imaging and Radiological Sciences, Chang Gung University, \\ 259 Wen Hua 1st Road, Kwei-Shan, Taoyuan 333323, Taiwan \\ ${ }^{5}$ National Space Organization (NSPO), 8F, 9 Prosperity 1st Road, Hsin-Chu City 300091, Taiwan
}

(Received June 24, 2021; accepted October 13, 2021; online published October 21, 2021)

Keywords: proton radiation, gamma radiation, soft error effect, total ionizing dose effect, MOSFET

Advances in semiconductor technology have enhanced the functionality of sensor arrays with reduced feature sizes. Owing to the spread of the Internet of Things, sensors can now be found in many applications operating in various environments. Proton and neutron radiation are always present around us but have not been detrimental to electronics at sea level. With the decreasing size of transistors and sensor elements and the increasing density of transistors in ICs of sensors and actuators, the effect of radiation on the reliability of semiconductor devices, sensors, and their electronic circuits (collectively called sensing systems) is no longer negligible, even at sea level. However, the knowledge of radiation physics and that of semiconductor physics are very different, and merging of the two sets of knowledge is necessary to evaluate the effect of radiation on the reliability of sensing systems. In this work, we summarize the extensive studies of the effects of radiation on semiconductor devices from space and avionics investigations, and we apply their results to study the radiation reliability of sensing systems in standard industry applications. In this work, we also illustrate how one can perform radiation reliability analysis for electronics in a proximity sensor, which we investigated by performing radiation experiments.

\section{Introduction}

Although radiation exists at sea level, until now its intensity has been too low to affect electronic circuits, and hence its effect has only been important for space applications. However, as the feature sizes of devices shrink with downscaling technology, the capacitance of the circuit nodes decreases accordingly. Consequently, the sensitivity of modern ICs to radiation is increasing. Furthermore, as the transistors become more closely packed, the severity of the radiation effect also increases, resulting in multiple-bit upsets instead of single-bit upsets being

${ }^{*}$ Corresponding author: e-mail: cmtan@cgu.edu.tw https://doi.org/10.18494/SAM.2022.3486 
more likely, as reported by Sun Microsystems, ${ }^{(1)}$ Texas Instruments, ${ }^{(2)}$ Virage Logic Corporation, ${ }^{(3)}$ Intel, ${ }^{(4,5)}$ Cypress Semiconductor, ${ }^{(6)}$ and IBM. ${ }^{(7)}$ It has been reported that singleevent upsets in avionics, high-end networks, and financial servers pose significant threats to the reliability of the corresponding systems. ${ }^{(8,9)}$ For example, Sun Microsystems encountered such an experience first-hand when some of its enterprise servers failed in the field because of soft errors in cache memory, which resulted in major customer dissatisfaction. ${ }^{(10,11)}$ Radiation indeed affects the reliable operation of electronic circuits and systems.

To illustrate the increasingly important effect of radiation on electronics, it was found that the critical charge, i.e., the charge that causes a flip in a bit of static random access memory (SRAM), decreases from $15 \mathrm{fC}$ at $250 \mathrm{~nm}$ technology to $1 \mathrm{fC}$ at $90 \mathrm{~nm}$ technology. If we use the soft error rate at $90 \mathrm{~nm}$ technology as a reference, the soft error rate will be 3.5 times higher for $22 \mathrm{~nm}$ technology, ${ }^{(12)}$ where the soft error rate is defined as the rate of occurrence of soft errors due to a nondestructive single-event transient, as will be introduced later.

Owing to the increasingly important effect of radiation on the reliability of electronics, including smart sensor networks containing advanced electronics, here we attempt to raise the awareness of including the radiation effect in the reliability evaluation of electronics through a brief description of the radiation effect on semiconductor devices and the typical tests carried out on their robustness to radiation. Examples of the degradation analysis of devices subjected to proton radiation will also be given. This work is intended to serve as a guide for the semiconductor industry.

\section{Materials and Methods}

There are three failure mechanisms of electronic circuits due to radiation: single-event transient (SET), total ionizing damage (TID), and displacement damage (DD). The thorough analysis of SET effects and the development of appropriate mitigation strategies have become key requirements in the design of fault-tolerant ICs and systems, and many semiconductor companies are now performing radiation tests on their advanced products.

\subsection{Electronics degradation mechanisms associated with radiation}

A SET (also called a SEE) is caused by the passage of a single energetic particle through or near a sensitive node of a circuit, creating electrical disorder that affects its normal operation. ${ }^{(13)}$ A densely ionized track is generated by the high-energy singly charged particle by depositing a sufficient amount of energy per unit path length to result in a destructive or nondestructive SET, depending on the response of the device and the amount of charge collected.(14) A nondestructive SET is also known as a soft error because it does not damage the device but causes a visible event or the corruption of output data or the data state. On the other hand, a destructive SET in a semiconductor device not only corrupts the data state but also permanently damages the device and hence it is known as a hard error. ${ }^{(15)}$ SETs dominate microelectronic reliability in the terrestrial environment. ${ }^{(16)}$ Most reliability failures are related to single-event upsets, namely, the flipping of digital bits in memories and sequential logic and the occasional single-event 
latch-up. ${ }^{(16)}$ Additionally, single-event burnout (SEB) in high-voltage power devices can be a reliability concern in the terrestrial environment. ${ }^{(17)}$

TID is mainly caused by the generation of defects in oxide layers due to incident energetic particles such as electrons, protons, and neutrons. In MOSFET devices, the ionizing radiation mainly affects the gate and field oxide layers, causing a shift in threshold voltage and an increase in leakage current. ${ }^{(18)}$ Ionizing radiation in MOSFET devices generates electron-hole pairs in the oxide, and as electrons have higher mobility, they are quickly swept away to the gate electrode under positive potential, while the lower-mobility holes are transported towards the $\mathrm{Si} / \mathrm{SiO}_{2}$ interface and trapped in the oxide or at the semiconductor/oxide interface. These trapped charges alter the threshold voltage of the MOSFET, resulting in a change in its electrical characteristics. ${ }^{(18-20)}$ TID is a significant issue for flash memories, power MOSFETs, and linear bipolar devices. ${ }^{(18)}$

When impinging high-energy radiation particles interact with the nuclei of the target material, atomic displacement or DD occurs. This type of nuclear interaction is quantified as non-ionizing energy loss. The parameters that are most sensitive to DD are minority carrier lifetime, diffusion length, carrier mobility, and carrier concentration. Since MOSFETs are majority carrier devices, they are inherently less sensitive to DD than minority carrier devices such as solar cells, diodes, linear bipolar devices, and charge-coupled devices. ${ }^{(21)}$ However, at smaller technology nodes, MOSFET devices may be sensitive to DD because the active region of the MOSFET device becomes comparable to the size of the DD cluster that can occur near the end of the range of a highly energetic particle. ${ }^{(14)}$ Fortunately, the occurrence of DD is generally rare as compared with SET and TID.

\subsection{Radiation environments for semiconductor devices and circuits}

There are two types of radiation environment: the natural environment, in which the radiation originates from only cosmic rays, and artificial radiation environments. For the natural environment, the dominant particles at sea level are neutrons, muons, protons, electrons, and pions generated from cosmic rays (mostly protons) incident on the earth's atmosphere. ${ }^{(22)}$ SEEs in microelectronic devices are mainly caused by neutrons at terrestrial altitudes and sea level because pions and muons have a very short half-life, and the Coulombic interactions can adequately attenuate electrons and protons. ${ }^{(16)}$

There are three factors that affect the neutron flux at any terrestrial location, of which altitude is the dominating factor. At flight altitudes of 28000-60000 feet, the neutron flux is 100 times higher than that at sea level. ${ }^{(23)}$ The second factor is latitude or geomagnetic rigidity, which is a function of geographical location. The magnetic fields emerging from the poles of the earth trap incoming cosmic ray protons within specific regions, leading to areas with high and low proton densities, which in turn lead to variations in terrestrial neutron flux as a function of geographical location. The neutron flux is higher in the equatorial region than at the poles because magnetic field lines at the poles are nearly perpendicular to the earth's surface, and the magnetic shielding effect or geomagnetic rigidity is minimized at the poles. ${ }^{(24)}$ The third factor is the solar activity cycle, which accounts for less than $30 \%$ of the neutron flux variation. ${ }^{(17)}$ 
Artificial radiation environments in which microelectronics might operate include medical, industrial, and defense applications. In medical applications, radiation exposure occurs most often from the use of diagnostic and treatment equipment such as X-ray and proton beam therapy machines. High doses of electron-beam (e-beam) or gamma-ray irradiation are also used for sterilizing surgical instruments and implantable electronics in operating rooms. There are numerous industrial uses of radiation. A wide range of applications rely on X-ray, gamma, and e-beam irradiation, including waste treatment, inspection, and security screening. Microelectronics are also exposed to doses of neutrons and gamma rays in high-radiation areas inside nuclear power plants. In defense environments, electronics must be hardened against brief but intense gamma-ray and neutron exposures, as well as against follow-on electromagnetic pulse effects from nuclear detonations. ${ }^{(17,25)}$

For microelectronics in most medical and industrial applications, TID is the primary radiation effect of concern, while in defense environments, concerns include SEEs, TID, DD, and promptdose (high dose rate) effects. ${ }^{(17)}$

\section{Reliability Tests for Radiation}

Although most reliability evaluations of products are carried out with an accelerated stress condition to shorten the test time, to evaluate the radiation reliability of semiconductor devices, care must be taken in the acceleration as there is a dose rate effect on the devices.

The effect of radiation on electronic devices depends on dose and dose rate effects. The dose effect results from the cumulative damage due to chronic radiation exposure, whereas the dose rate effect originates from the deposition of an extremely high dose over a short time. The dose effect has two main categories, TID and DD, as mentioned earlier. Degradation due to TID has an additional complication known as enhanced low-dose-rate sensitivity (ELDRS), which primarily affects bipolar devices. In the case of ELDRS, bipolar devices show more noticeable DC parameter degradation. This is characterized by a low-dose-rate enhancement factor (EF), defined quantitatively as the ratio of the parametric degradation at a low dose rate (LDR) $[<360$ $\mathrm{rad}(\mathrm{Si}) / \mathrm{h}$ ] to that at a standard dose rate $[3.6$ to $36 \mathrm{krad}(\mathrm{Si}) / \mathrm{h}$ ] for a fixed dose level. However, the EF is not a universal value, and it varies within the same part for different parameters and part types. ${ }^{(26)}$ It is important to know whether a device has ELDRS because the actual dose rate encountered in most radiation environments including space environments is very low. Pershenkov et al. studied the physical mechanism of the ELDRS effect in bipolar operational amplifiers for a wide range of dose rates and found that the accumulation of defects during LDR irradiation depends on the irradiation time only, while the contribution of true dose rate effects to the enhanced LDR sensitivity effect is related to processes during post-irradiation annealing. ${ }^{(27)}$ Consequently, tests performed at a high dose rate to shorten the test time may not be feasible for extrapolating to a field condition where the dose rate is low, and this is especially true for bipolar devices.

To study the risk associated with electronic devices to be used in space, terrestrial, or artificial radiation environments, devices must be tested under real radiation conditions. Table 1 summarizes the different tests and their purpose for electronic devices. ${ }^{(28)}$ 
Table 1

Summary of radiation tests for electronic devices.

\begin{tabular}{|c|c|c|}
\hline Test & Type of product & Purpose \\
\hline High dose rate (HDR) ${ }^{(29)}$ & $\begin{array}{l}\text { Without ELDRS or time- } \\
\text { dependent effects (TDEs) }\end{array}$ & Radiation lot acceptance test (RLAT) \\
\hline $\begin{array}{l}\text { Metal oxide semiconductor } \\
\text { accelerated anneal test (MAAT) }\end{array}$ & MOS devices & To determine TDEs in technology \\
\hline Room temperature anneal test ${ }^{(30)}$ & Devices that fail HDR test & $\begin{array}{l}\text { To determine whether product } \\
\text { qualifies for LDR test }\end{array}$ \\
\hline ELDRS characterization $^{(31)}$ & Bipolar linear elements & To determine ELDRS effect \\
\hline $\mathrm{LDR}^{(32)}$ & Devices without ELDRS & Alternative of HDR for RLAT \\
\hline LDR+1.5x over test ${ }^{(28)}$ & Devices with ELDRS & RLAT \\
\hline Accelerated ELDRS ${ }^{(33)}$ & Bipolar linear elements & $\begin{array}{c}\text { Alternative RLAT verified through } \\
\text { characterization }\end{array}$ \\
\hline SEB and SEGR ${ }^{(34,35)}$ & Power MOSFETs & To study effect of single particle \\
\hline $\mathrm{SEE}^{(36)}$ & Digital devices & To study effect of single particle \\
\hline
\end{tabular}

These tests are performed in specially designed laboratories to ensure that the tests meet the requirements of measurement standards, reliability, and safety. Several test standards have been published for different radiation effects. To test the TID effect, MIL-STD-883 Test Method (TM) 1019 standards are used, which describe the test and qualification using a Co-60 source. TM 1019 provides flexible test options at different dose rates based on semiconductor technology TID sensitivity.

To determine the time-dependent effects in MOSFET structures, the MOSFET accelerated anneal test (MAAT), also known as the rebound test, is performed. For many applications, the radiation environment has an LDR, and for such environments, the LDR test based on TM 1019 specifications is performed. The test range of TM 1019 was restricted to the range of 50 to 300 $\mathrm{rad} / \mathrm{s}$ until 1991, after which it was updated to below $50 \mathrm{rad} / \mathrm{s}$.

The ELDRS test specification is provided in TM 1019; however, it does not provide any information about the sample size nor on the number of devices under test (DUTs) to use for product qualification. The test methods for discrete transistors for military and space applications are given in MIL-STD-750, which includes TM 1080 for SEB and single-event gate rupture (SEGR) for the testing of power MOSFETs.

For radiation testing, the DUTs must be biased under the worst operating condition so that the maximum drift in the parameters of the DUT can be observed. In some cases, in situ measurement is performed, which requires real-time monitoring and characterization equipment such as mixed signal oscilloscopes, source meters, a semiconductor characterization system, and arbitrary function generators. To irradiate DUTs with proton or neutrons, proton and neutron generators are used, respectively. The neutron generators commonly used in laboratories are compact linear particle accelerators, whereas protons are generated from either cyclotrons or synchrotrons. Figure 1 shows the cyclotron-based proton generator used in this work, which is in the Particle Physics and Beam Delivery Core Laboratory at the Proton and Radiation Therapy Center of Chang Gung Memorial Hospital, Linkou, Taiwan. 


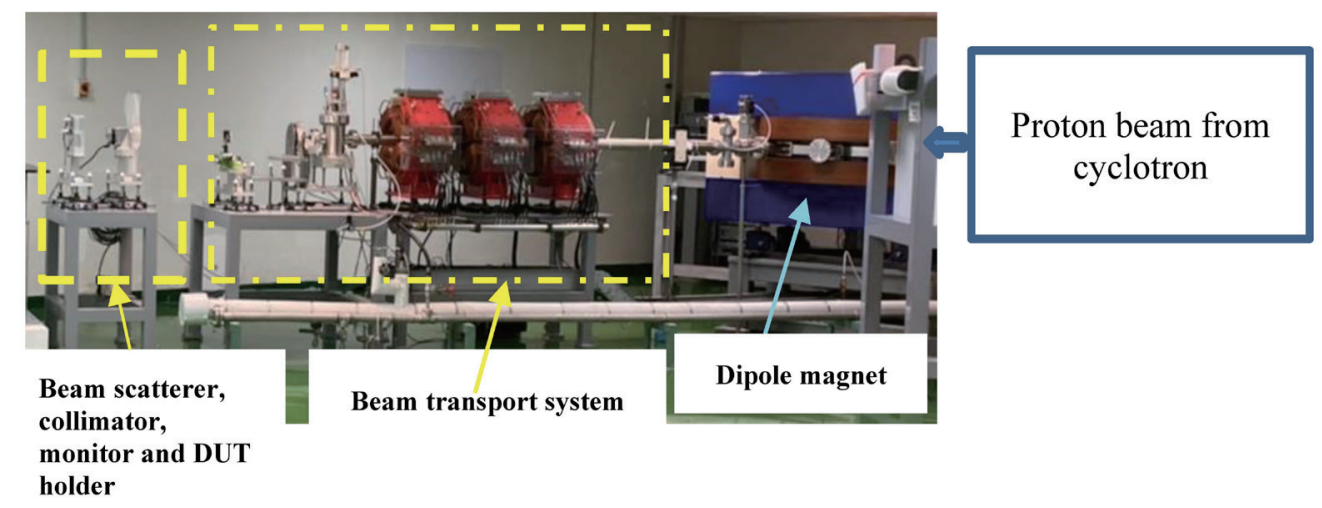

Fig. 1. (Color online) Proton beam test setup for soft error rate estimation.

The cyclotron generates a static $230 \mathrm{MeV}$ proton beam, which passes through an energy selection system consisting of an energy degrader and a series of devices to restrict the beam size and energy dispersion so that the desired test energy can be obtained. The selected energy of the proton beam is transported to the DUT through the beam transport system, which has a series of magnets to guide the moving proton beam. The position and size of the beam are monitored using a beam monitor device.

\section{Experimentation}

Both electronic devices and circuit boards are subjected to proton radiation tests with different proton energies. Units or boards are powered up during testing to monitor their performance. Figure 2 shows the soft error rate test setup for a proton test, where the $200 \mathrm{MeV}$ proton scanning beam in Chang Gung Memorial Hospital at Linkou is used. The fluence is set to $1 \times 10^{11}$ proton $/ \mathrm{cm}^{2}$. The TID test is carried out using Co-60 radiation at $20 \mathrm{krad}$ in National Tsinghua University, Taiwan.

For the soft error rate evaluation, an IC with various functions is tested under a proton beam with the setup shown in Fig. 2, and the number of soft error events is counted, where the counts are classified according to the function.

For TID/DD evaluation, the DUT is a proximity sensor, which contains a MOSFET switch whose specifications we cannot disclose for reasons of confidentiality. This MOSFET device is housed in a miniature cylinder with dimensions of $\varnothing 5 \mathrm{~mm} \times 26 \mathrm{~mm}$. The switch is connected during the test as shown in Fig. 3. A $24 \mathrm{~V}$ power supply is connected to the switch in series with a load resistor. 


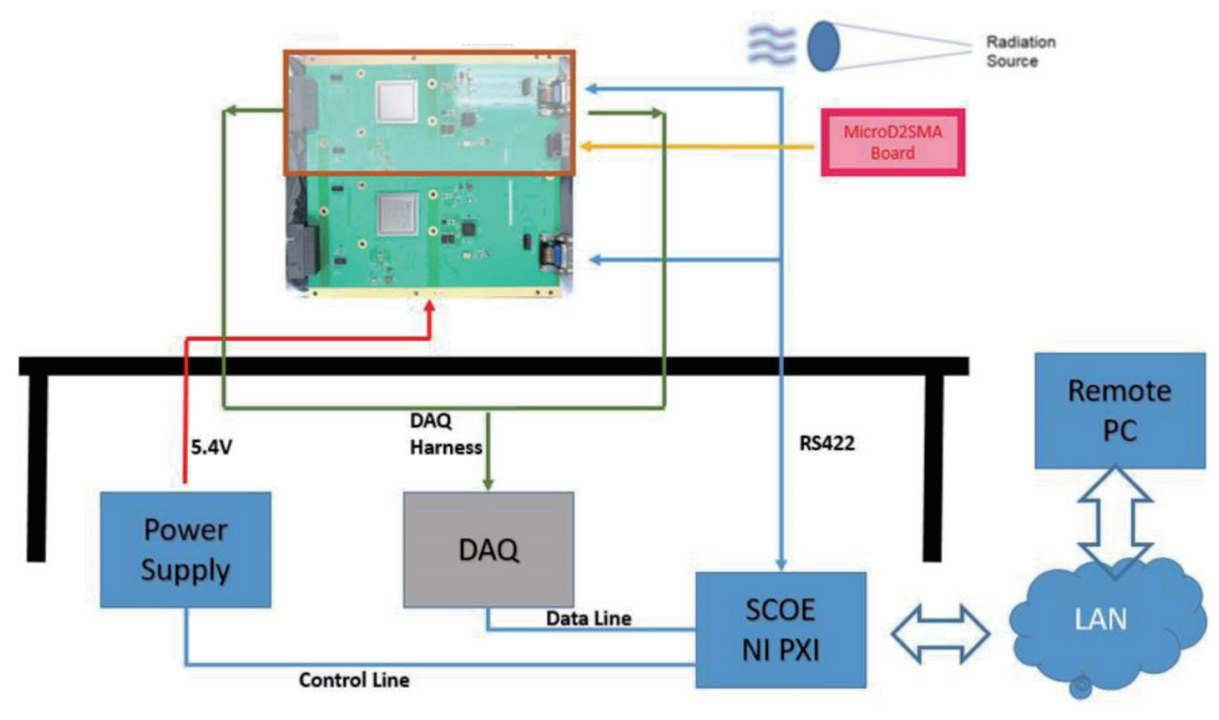

Fig. 2. (Color online) Setup for determining proton soft error rate.

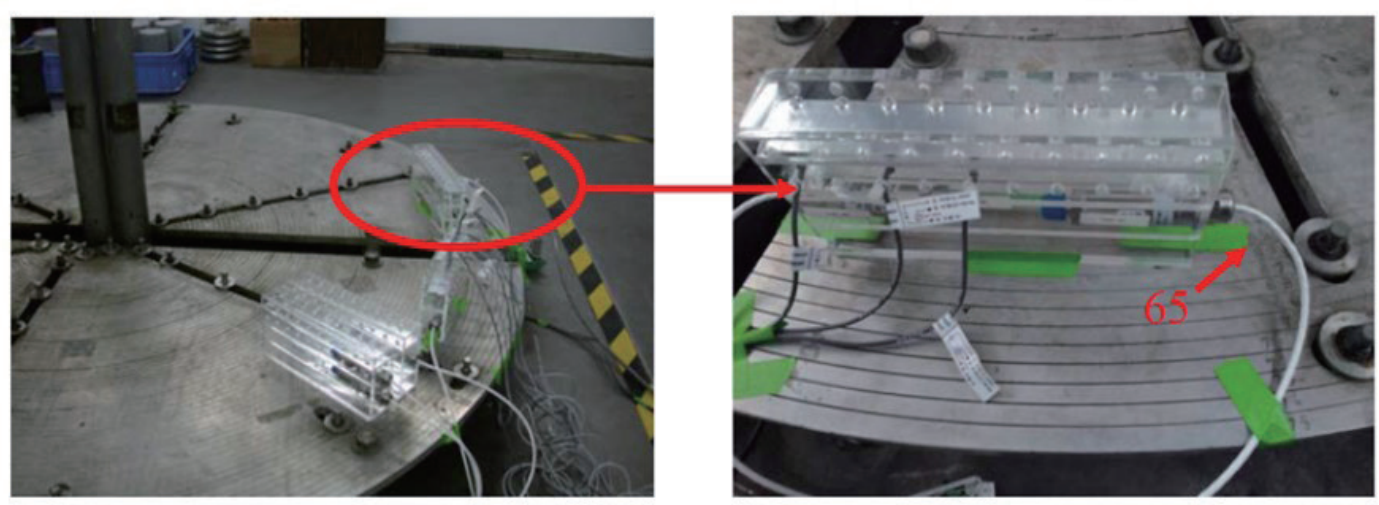

Fig. 3. (Color online) Test setup for proximity switch.

\section{Experimental Results and Analysis}

\subsection{Soft error rate}

The soft error rate can be computed using the following equation: ${ }^{(37,38)}$

$$
\text { SEEs }=\sum \text { Cross section }(E) \times F l u x(E),
$$

where Flux $(E)$ can be calculated via Crème $96,{ }^{(39,40)}$ and the cross section at different radiation energies $(E)$ can be calculated via the following models: 
- Weibull

- Bendel two-parameter

- Bendel one-parameter

Although the Weibull model is most accurate, it contains four parameters, and thus radiation experiments are performed at four different energies, which can be very time-consuming and costly. The Bendel two-parameter model also requires experiments to be carried out at two different energies, and it is thus not commonly employed. In this work, the Bendel one-parameter model is used for the computation of the soft error rate.

Table 2 shows our proton test results for a device with several functions. The number of soft errors occurring at a given proton energy $E$ is recorded, from which the soft error rate is obtained. Using Eq. (1) and the known value of the proton flux set in the experiment, we can compute the cross section at proton energy $E$ set in the experiment. Using the following Bendel one-parameter model, we can determine the value of the parameter $A$ :

$$
F(E)=(B / A)^{14}\left[1-\exp \left(-0.18 y^{1 / 2}\right)\right]^{4},
$$

where $F(E)$ is the cross section at energy $E$ and

$$
y(E)=(E-A)(18 / A)^{1 / 2} \quad \text { if } E>A,
$$

with $A$ and $B$ as device-specific parameters. The computed values of $A$ for the different functions are shown in Table 2. The empirical value of $B$ for most components is 24 .

Once the values of $A$ and $B$ are determined, one can compute the soft error rate under various radiation environments using the radiation energy spectrum given by a database such as Crème 96 and obtain the total soft error rate using Eq. (1). Table 2 shows the SEE/day obtained using the Crème 96 database for the application of the DUT in a low-earth orbit.

\subsection{TID and DD analyses}

Figure 4 shows the load current over time during the test. A small decrease in the load current is observed. As the supplied voltage is held constant at $24 \mathrm{~V}$, the observed decrease in current indicates an increase in the switch resistance. By taking unit $\# 2$ as an example, the change in current $(\Delta I)$ is from 0.01255 to $0.0125 \mathrm{~A}$, a small decrease of $0.00005 \mathrm{~A}$. However, this small

Table 2

Experimental test and computation data of a device from our proton test to its application in the low-earth orbit environment.

\begin{tabular}{lccll}
\hline DUT & SEEs & Cross section & \multicolumn{1}{c}{$A$} & SEE/day \\
\hline Function 1 & 26 & $1.51 \mathrm{E}+02$ & 16.771 & 0.001493 \\
Function 2 & 28 & $1.63 \mathrm{E}+02$ & 16.68 & 0.001615 \\
Function 3 & 31 & $1.80 \mathrm{E}+02$ & 16.562 & 0.00179 \\
Function 4 & 1 & $5.81 \mathrm{E}+00$ & 21.1654 & $5.15 \mathrm{E}-05$ \\
Function 5 & 27 & $1.57 \mathrm{E}+02$ & 16.725 & 0.001554 \\
\hline
\end{tabular}




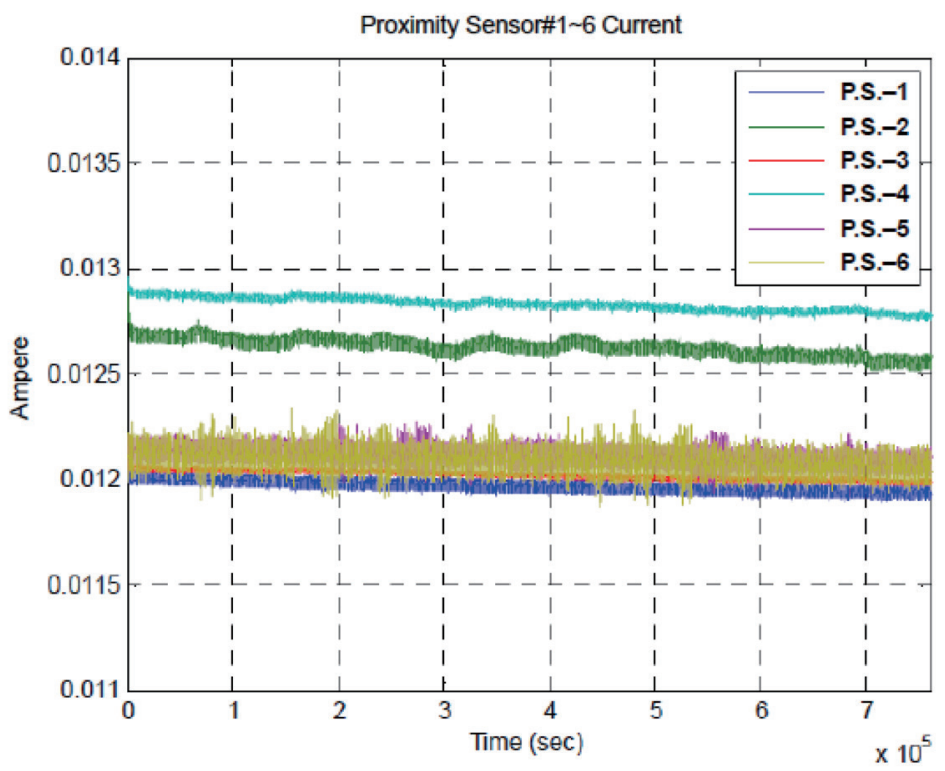

Fig. 4. (Color online) Variation of load current during Co-60 test on six proximity switches as DUTs.

change in current may not be small for the corresponding change in switch resistance, as illustrated by Eq. (4), where we assume that the switch resistance is $R$ before radiation and $R$ ' after radiation.

$$
\Delta I(i n \%)=\frac{\frac{24}{R^{\prime}+2 K}-\frac{24}{R+2 K}}{\frac{24}{R+2 K}}=\frac{\Delta R}{2 K} \quad \text { if } R, R^{\prime} \ll 2 K
$$

Substituting $\Delta I=0.4 \%$, we have $\Delta R=8 \Omega$. This is a substantial change in resistance for an electronic switch. The seemingly small change is masked by the $2 \mathrm{k} \Omega$ resistance connected during testing.

The test is performed at room temperature for a duration of less than a year, with a test current of around $0.012 \mathrm{~A}$. This is well within the specification of $200 \mathrm{~mA}$ for the switch; thus, large degradation due to the applied current stress is unlikely in this test. We can thus conclude that such degradation is due to the Co-60 TID effect in the oxide layer, where electron-hole pairs are generated by the Co-60 gamma radiation. ${ }^{(34,41,42)}$ No degradation occurs in the substrate or conducting channel because the pin-to-pin (PP) resistance without a load showed no change for all six DUTs.

We also observe that the current becomes very noisy for units \#3 and \#6. This is a typical signature of telegraph noise in an oxide due to charge trapping in the oxide. ${ }^{(43)}$

It is interesting that the six DUTs showed no change in the PP resistance immediately after the radiation test. However, when the same measurement was carried out five days later with the load connected, five DUTs failed, and the sixth showed a large change in the load current upon 
remeasurement. This is because, in addition to oxide traps, radiation also leads to the formation of interface traps at the $\mathrm{Si} / \mathrm{SiO}_{2}$ interface. Interface traps exist within the silicon band gap at the interface. When electron-hole pairs are generated by the radiation, electrons can move quickly through the oxide to the gate electrode and some of them recombine with holes during the movement. Owing to the resulting absence of electrons in the oxide, the holes that diffuse slowly through hopping toward the interface are trapped at the interface, causing the build-up of interface-trap charge. It is also believed that hydrogen ions in the oxide are likely released as holes that "hop" through the oxide or are trapped near the $\mathrm{Si} / \mathrm{SiO}_{2}$ interface. The hydrogen ions can also drift to the $\mathrm{Si} / \mathrm{SiO}_{2}$ interface, where they may react to form interface traps. The build-up of interface-trap charge can take hours to saturate after a pulse of ionizing radiation. ${ }^{(44)}$ Therefore, the passing but subsequent failure of DUTs is due to such build-up of interface-trap charge.

In summary, from the analysis of the degradation pattern, we can conclude that all the switches are degraded by TID. Electron-hole pair generation in the oxide is the prime reason for all the observed TID effects. As the PP resistances of the switches remain unchanged, it can be concluded that there is no DD.

\section{Conclusions}

Radiation is becoming a new environmental stress factor for advanced semiconductor devices and their associated circuits, and its effect is no longer limited to applications in space and avionics. These devices and circuits can also be part of a smart sensor network. The effect of radiation on electronic devices at sea level has been reported. In this work, we presented a brief description of the three main radiation effects on these devices, namely, the soft error rate, total ionizing dose, and displacement damage. Experiments were performed on some electronic devices, and the computation of the soft error rate and an investigation to determine the presence of total ionizing dose and displacement damage were demonstrated. These results show examples of the radiation reliability evaluation of electronic devices, and areas where care must be taken in the use of accelerated radiation tests for such evaluation were also noted.

\section{Acknowledgments}

The authors would like to express their gratitude to Dr. Steven Chen, principal investigator of the FORMOSAT-8 program at NSPO, for giving them the opportunity to collaborate with each other. They would also like to thank the NSPO Reaction Control Subsystem (RCS) team for its enormous help in promptly providing valuable radiation test data.

\section{References}

1 C. W. Slayman: IEEE Trans. Device Mater. Reliab. 5 (2005) 397. https://doi.org/10.1109/TDMR.2005.856487

2 R. Baumann: IEEE Design Test Comput. 22 (2005) 258. https://doi.org/10.1109/MDT.2005.69

3 N. Derhacobian, V. A. Vardanian, and Y. Zorian: Records of the IEEE Int. Workshop on Memory Technology, Design and Testing (IEEE, 2004) 104-110. https://doi.org/10.1109/MTDT.2004.1327992 
4 M. Spica and T. M. Mak: Records of the IEEE Int. Workshop on Memory Technology, Design and Testing (IEEE, 2004) 111-116. https://doi.org/10.1109/MTDT.2004.1327993

5 J. Maiz, S. Hareland, K. Zhang, and P. Armstrong: IEEE Int. Electron Devices Meeting (IEEE, 2003) 519-522. https://doi.org/10.1109/IEDM.2003.1269335

6 D. Radaelli, H. Puchner, S. Wong, and S. Daniel: IEEE Trans. Nucl. Sci. 52 (2005) 2433. https://doi.org/10.1109/ TNS.2005.860675

7 P. J. Meaney, S. B. Swaney, P. N. Sanda, and L. Spainhower: IEEE Trans. Device Mater. Reliab. 5 (2005)419. https://doi.org/10.1109/TDMR.2005.859577

8 S. Mitra, N. Seifert, M. Zhang, Q. Shi, and K. S. Kim: Computers 38 (2005) 43. https://doi.org/10.1109/ MC. 2005.70

9 E. Normand: IEEE Trans. Nucl. Sci. 43 (1996) 2742. https://doi.org/10.1109/23.556861

10 Embedded SRAM Test and Repair Moves On-chip: https://www.design-reuse.com/news/572/embedded-sramtest-repair-moves-chip.html (accessed March 2021).

11 Soft Memory Errors and Their Effect on Sun FireTM Systems: https://docs.oracle.com/cd/E19095-01/sf4810. srvr/816-5053-10/816-5053-10.pdf (accessed March 2021).

12 E. Ibe, T. Hitoshi, Y. Yahagi, Ken-ichi, Shimbo, and T. Toba: 3rd Workshop on Dependable and Secure Nanocomputing (2009).

13 Overview of Single Event Effects: https://www.inaoep.mx/seressa2015/archivos/Lunes 16 30\%20\%20 Buchner.pdf (accessed May 2021).

14 D. M. Fleetwood: IEEE Trans. Nucl. Sci. 68 (2021) 509. https://doi.org/10.1109/TNS.2021.3053424

15 F. W. Sexton: IEEE Trans. Nucl. Sci. 50 (2003) 603. https://doi.org/10.1109/TNS.2003.813137

16 Landmarks in Terrestrial Single-Event Effects 2013 NSREC Short Course: http://www-vlsi.es.kit.ac.jp/ SERconf/2014/Baumanall.pdf (accessed May 2021).

17 R. Baumann and K. Kruckmeyer: Radiation Handbook for Electronics (Texas Instruments, 2019) pp. 4-67.

18 D. M Fleetwood: IEEE Trans. Nucl. Sci. 65 (2018) 1465. https://doi.org/10.1109/TNS.2017.2786140

19 H. J. Barnaby: IEEE Trans. Nucl. Sci. 53 (2006) 3103. https://doi.org/10.1109/TNS.2006.885952

20 T. R. Oldham and F. B. McLean: IEEE Trans. Nucl. Sci. 50 (2003) 483. https://doi.org/10.1109/TNS.2003.812927

21 E. E. Allam, C. Inguimbert, A. Meulenberg, A. Jorio, and I. Zorkani: J. Appl. Phys. 123 (2018) 95703. https:// doi.org/10.1063/1.5013211

22 J. F. Ziegler: IBM J. Res. Dev. 40 (1996) 19. https://doi.org/10.1147/rd.401.0019

23 E. Normand: IEEE Trans. Nucl. Sci. 43 (1996) 461. https://doi.org/10.1109/23.490893

24 Ionizing Radiation and Radiation Safety in Aerospace Environments: https://trid.trb.org/view/1862928 (accessed April 2021).

25 W. A. Smit and H. J. Hutter: SIPRI Workshop on Measures to Reduce the Dangers of Surprise Attack in Europe (1983) 1-15.

26 D. Núñez, M. Poizat, J. Jiménez, E. Muñoz, and M. Domínguez: 2014 IEEE Radiation Effects Data Workshop (IEEE, 2014) 1-6. https://doi.org/10.1109/REDW.2014.7004568

27 V. S. Pershenkov, A. S. Petrov, A. S. Bakerenkov, V. N. Ulimov, V. A. Felytsyn, A. S. Rodin, V. V. Bely-akov, V.A. Telets, and V. V. Shurenkov: Microelectron. Reliab. 76-77 (2017) 703. https://doi.org/10.1016/j. microrel.2017.07.025

28 Test Method 1019 (Total Ionizing Dose) Its time for 1019.10: https://s3vi.ndc.nasa.gov/ssri-kb/static/ resources/1140\%20-\%20Turflinger\%20-\%20NEPP\%202019\%20TM1019\%20Rewrite\%20Final.pdf (accessed May 2021).

29 Department of Defense Test Method Standard MIL-STD 883 (Microcircuits): http://scipp.ucsc.edu/groups/ fermi/electronics/mil-std-883.pdf (accessed May 2021).

30 Room Temperature Anneal Test: http://reliabilityanalytics.com/reliability engineering library/MIL-STD$883 \mathrm{H}$ Test_Method_Standard_Microcircuits_26 Feb_2010/MIL-STD-883H Test_Method_Standard Microcircuits_26_Feb_2010_pp_126.pdf (accessed May 2021).

31 R. D. Harris, S. S. McClure, B. G. Rax, D. O. Thorbourn, A. J. Kenna, K. B. Clark, and T. Y. Yan: 2010 IEEE Radiation Effects Data Workshop (IEEE, 2010) 104-110. https://doi.org/10.1109/REDW.2010.5619505

32 Low Dose Rate Acceptance Testing: https:/www.renesas.com/us/en/application/technologies/radiationhardened-solutions/low-dose-rate-acceptance-testing-eldrs (accessed May 2021).

33 X. Wang, W. Lu, S. Yao, X.L. Li, Q. Guo, C.F. He, X. Yu, and J. Sun: 18th European Conf. Radiation and Its Effects on Components and Systems (RADECS 2018) 1-4. https://doi.org/10.1109/RADECS45761.2018.9328666

34 Radiation Hardness Assurance Testing of Microelectronic Devices and Integrated Circuits: Test Guideline for Proton and Heavy Ion Single-Event Effects: https://citeseerx.ist.psu.edu/viewdoc/ download?doi=10.1.1.613.7439\&rep=rep1\&type=pdf (accessed January 2021). 
35 Testing Guideline for Single Event Gate Rupture (SEGR) of Power MOSFETs: https://nepp.nasa.gov/ files/16408/08 163 4_JPL_Scheick.pdf (accessed January 2021).

36 Test Procedure for the Management of Single-Event Effects in Semiconductor Devices from Heavy Ion Irradiation: https://www.jedec.org/standards-documents/docs/jesd-57 (accessed January 2021).

37 E. L. Petersen, J. C. Pickel, J. H. Adams, and E. C. Smith: IEEE Trans. Nucl. Sci. 39 (1992) 1577. https://doi. org $/ 10.1109 / 23.211340$

38 E. Petersen: Single Event Effects in Aerospace (John Wiley \& Sons, 2012) 1st ed., pp. 251-303.

39 P. R. Boberg, B. Brownstein, W. F. Dietrich, E. O. Flueckiger, E. L. Petersen, M. A. Shea, D. F. Smart, and E.C. Smith: IEEE Trans. Nucl. Sci. 44 (1997) 2150. https://doi.org/10.1109/23.659030

40 How to Run CREME96 - CREME-MC site: https://creme.isde.vanderbilt.edu/CREME-MC/help/how-to-runcreme96 (accessed April 2021).

41 J. Schwank, M. R. Shaneyfelt. D. M. Fleetwood, J. A. Felix, P. E. Dodd, P. Paillet, and V. Véronique FerletCavrois: IEEE Trans. Nucl. Sci. 55 (2008) 1833. https://doi.org/10.1109/TNS.2008.2001040

42 S. Stanković, D. Nikolić, N. Kržanović, L. Nadjdjerdj, and V. Davidović: IEEE 31st Int. Conf. Microelectronics (IEEE, 2019) 181-184. https://doi.org/10.1109/MIEL.2019.8889613

43 T. Grasser: Microelectron. Reliab. 52 (2012) 39. https://doi.org/10.1016/j.microrel.2011.09.002

44 Radiation Effects in MOS Oxides: https://www.osti.gov/servlets/purl/1147853 (accessed June 2021).

\section{About the Authors}

Cher Ming Tan received his Ph.D. degree in electrical engineering from the University of Toronto in 1992. He had 10 years of experience working in reliability in the electronic industry before joining Nanyang Technological University (NTU) as a faculty member in 1996. In 2014, he joined Chang Gung University, Taiwan, and set up the Research Center on Reliability Sciences and Technologies in Taiwan, for which he acts as center director. He is also a professor in the Electronic Engineering Department of Chang Gung University and an honorary chair professor in Ming Chi University of Technology, Taiwan. He has published 300+ international journal and conference papers, and given 10+ keynote talks, 50+ invited talks, and several tutorials in international conferences. He has written five books and three book chapters in the field of reliability. He has been an editor and associate editor of several scientific journals. He is also on the committee of IEEE EDS Reliability Physics. He has been a chair and a founding chair of various societies on reliability, was a recipient of the IEEE Region 10 Outstanding Volunteer Award in 2011, and the first individual recipient of the Ishikawa-Kano Quality Award in Singapore in 2014. His research interests include modeling the reliability and failure physics of electronic components and systems; finite element modeling of material degradation; statistical modeling of engineering systems, nanomaterials, and device reliability; and the prognosis and health management of engineering systems. For more details, please visit www. chermingtan.com (cmtan@cgu.edu.tw)

Vimal Kant Pandey received his B.Sc. degree in physics and maths from St. Andrew's College, Gorakhpur, India, in 2006, his M.Sc. degree in electronics from Bundelkhand University, Jhansi, India, in 2008, and his M.Tech. degree in VLSI design from Guru Gobind Singh Indrapastha University, Delhi, India, in 2010. He received his Ph.D. degree in electronic engineering from the Department of Electronic Engineering, Chang Gung University, Taoyuan, Taiwan, in 2021. He was a research scholar with the Center for Reliability Sciences and Technologies, Chang 
Gung University, until 2021, and he is now a project leader in MOXA, Inc. His research interests include analog ICs, radiation effects on electronic devices, GaN-based circuit design for radiation detection, and signal processing for proton beam therapy.

Yueh Chiang received his B.Sc. degree from National Taipei University and his Ph.D. degree in medical imaging and radiological sciences from Chang Gung University in 2021. He was a research assistant with the Medical Physics Laboratory in Chang Gung University from 2015 to 2021. Upon graduation, he worked as a principal engineer with the Digital IPs Solution Division in Taiwan Semiconductor Manufacturing Company, Ltd. His research interests include medical physics, particle physics, single-event effects, the total ionization effect, radiation protection, and Monte Carlo simulation.

Tsung Ping Lee received his master's degree in computer engineering from National Chiao Tung University, Taiwan, in 2006. He is currently working for the National Space Organization (NSPO) in product-assurance-related work, including reliability prediction, material selection, workmanship assurance, EEE parts inspection, radiation analysis, and units and satellites testing. 